\title{
Análise de Sentimentos sobre o lockdown durante a pandemia de COVID-19: o caso brasileiro
}

\author{
Paulo Alves da Silva1 ${ }^{1}$, Felipe A. Leite ${ }^{1}$, Douglas Vitório ${ }^{1}$, Marcelo Iury S . Oliveira ${ }^{1}$ \\ ${ }^{1}$ Unidade Acadêmica de Serra Talhada - Universidade Federal Rural de Pernambuco \\ (UAST - UFRPE) - Caixa Postal 063 - 56.900-000 - Serra Talhada - PE - Brasil \\ \{alvespaulo737, lipe6330, douglas.alisson17, marceloiury\}@gmail.com
}

\begin{abstract}
The high contamination risk of COVID-19 has led to several countries taking social distance and isolation measures. Lockdown is the highest level of social distance measure. Although the lockdown in an excellent tool for health protection, the adoption of this measure divides opinions. In this sense, a Sentiment Analysis appears as a mean that can be used to understand the population opinion about lockdown. Thus, the present work carry out a Sentiment Analysis in order to understand how the Brazilian population is reacting in relation to the lockdown implementation during the COVID-19 pandemic.
\end{abstract}

Resumo. O alto risco de contaminação do COVID-19 tem levado vários países a tomar medidas de distanciamento social e isolamento. O Lockdown é o a medida mais rígida de distanciamento social. Embora o lockdown seja uma excelente ferramenta para a proteção da saúde, a adoção dessa medida divide opiniões. Nesse sentido, a Análise de Sentimento surge como um meio que pode ser utilizado para compreender a opinião da população sobre o lockdown. Assim, o presente trabalho realiza uma Análise de Sentimento com o objetivo de compreender como a população brasileira está reagindo em relação à implantação do lockdown durante a pandemia do COVID-19.

\section{Introdução}

O vírus da Síndrome Respiratória Aguda Grave 2 (SARS-CoV-2), conhecido como "novo Coronavírus", mudou completamente a rotina das pessoas em função do seu alto nível de contágio e taxa de mortalidade não desprezível [Moreira 2020, Xavier et al. 2020]. No início da pandemia, em função do escasso conhecimento sobre os modos de transmissão e o papel dos portadores assintomáticos na difusão do SARS-CoV-2, aliado à inexistência de vacinas e alternativas terapêuticas específicas [Carvalho et al. 2020, Xavier et al. 2020], vários governos ao redor do mundo decretaram medidas de distanciamento social como forma de conter o avanço do contágio.

Entre as medidas mais restritivas de distanciamento social está o lockdown, o qual impõe limitações de circulação, restringe o funcionamento de escolas e negócios, bem como estabelece a proibição de aglomerações. Esse é o nível mais alto de segurança e pode ser necessário em situação de grave ameaça ao sistema de saúde [Bai et al. 2020]. O lockdown é considerado eficaz para redução da curva de casos e permite a reorganização do sistema de saúde em situação de aceleração descontrolada de casos e óbitos ${ }^{1}$. Contudo, lockdowns não são isentos de custos, riscos ou danos. Segundo [Bai et al. 2020], a

\footnotetext{
${ }^{1}$ http://www.cofen.gov.br/wp-content/uploads/2020/04/Boletim-07-MS-06-04-2020.pdf.pdf
} 
imposição de lockdowns durante a pandemia tem apresentado consequências econômicas, sociais e emocionais negativas na população. Assim, a adoção dessa medida ainda divide opiniões, tanto entre a população geral quanto entre estudiosos [Watson et al. 2020].

De forma a compreender melhor o sentimento dos brasileiros a respeito da adoção de lockdowns, este trabalho realizou um estudo de análise de sentimentos das mensagens da rede social Twitter. Segundo [Liu and Zhang 2012], a Análise de Sentimentos (AS), também chamada de Mineração de Opinião, é a área de estudo que analisa os sentimentos e opiniões das pessoas acerca de entidades. Estas entidades podem ser produtos, serviços, organizações, indivíduos, problemas, eventos, tópicos e seus atributos; e as opiniões são expressas de forma não-estruturada, como em texto, por exemplo. A análise de sentimento geralmente consiste em classificar uma opinião em um dentre três rótulos: positiva, negativa ou neutra [Liu and Zhang 2012, Yue et al. 2018, Alswaidan and Menai 2020].

Com esta análise, pretende-se mostrar como os usuários brasileiros do Twitter que emitiram mensagens sobre o tema lockdown reagiram em relação às decisões tomadas no sentido da implantação da medida no país. O trabalho também visa determinar se fatores geográficos, econômicos e ideológico-partidários exercem influência no posicionamento destes usuários.

O restante deste trabalho está organizado da seguinte forma: a Seção 2 apresenta os trabalhos relacionados; a Seção 3 detalha a metodologia utilizada nesta pesquisa; enquanto que os resultados encontrados e suas discussões podem ser encontrados na Seção 4; e, por fim, a Seção 5 traz as considerações finais.

\section{Trabalhos Relacionados}

A análise de sentimentos nas redes sociais tornou-se um grande tema de estudo [Yue et al. 2018, Alswaidan and Menai 2020]. Um número crescente de trabalhos têm apresentado ou novas técnicas de análise de sentimentos ou estudos que analisam o sentimento de pessoas a respeito de temas específicos, como a aceitação de políticas públicas [Yue et al. 2018, Alswaidan and Menai 2020]. Inclusive, o emprego da análise de sentimentos a respeito do lockdown durante o período pandêmico já foi abordado também pela literatura, tais como os trabalhos de [Barkur et al. 2020, Kaur and Ranjan 2020, Chehal et al. 2020, Pastor 2020, Dubey 2020].

Em [Barkur et al. 2020], os autores realizaram uma Análise de Sentimentos após a determinação de um lockdown na Índia, durante a pandemia de COVID-19. Ao realizar o processamento dos dados, eles constataram que havia um número pequeno de tweets expressando emoções como surpresa, tristeza, preocupação, raiva, negatividade, medo e repulsa, significando que, de maneira geral, a população indiana reagiu bem às medidas tomadas. Também foi salientado que sentimentos positivos, como o de confiança, se destacaram na nuvem de palavras. Entretanto, pôde-se verificar nesse trabalho a falta de detalhamento dos recursos utilizados para sua elaboração, bem como o período de coleta dos tweets, que foi relativamente curto.

O trabalho de [Kaur and Ranjan 2020] também teve como objetivo realizar uma Análise de Sentimentos sobre o lockdown vivenciado na Índia durante a pandemia, observando uma predominância de sentimentos positivos. Os autores, contudo, não deixaram evidente quais algoritmos foram utilizados para obtenção dos resultados, como também 
não apresentaram justificativas que poderiam ter levado a população indiana a se expressar a favor ou contra a medida.

[Chehal et al. 2020] também utilizaram a Índia como objeto de estudo. Porém, diferentemente de [Barkur et al. 2020] e [Kaur and Ranjan 2020], os autores realizaram a Análise de Sentimentos dos indianos a partir de tweets coletados acerca de lockdowns ocorridos em dois períodos de tempo diferentes, denominados Lockdown 2.0 e Lockdown 3.0. Comparando sentimentos e emoções nos dois lockdowns, eles concluíram que emoções como desgosto, tristeza e antecipação apresentaram um aumento considerável durante o Lockdown 3.0 em relação ao Lockdown 2.0. No entanto, vale ressaltar que o estudo não apresentou os fatores que levaram a população a se manifestar dessa forma.

Em seu trabalho, [Pastor 2020], a autora teve como objetivo identificar por meio da Análise de Sentimentos como a população das Filipinas reagiu em relação à COVID19 e às decisões de implantação de uma quarentena no país. Ela concluiu que, na maior parte dos tweets analisados, havia uma grande margem de diferença entre os sentimentos positivo e negativo, tendo este último sido predominante. No trabalho houve, porém, a ausência de um estudo dos fatores que influenciaram a população a se expressar dessa maneira. Além de que a autora não apresentou as emoções em forma de nuvens de palavras, o que poderia ter auxiliado na identificação desses fatores.

Por fim, [Dubey 2020] aplicou a Análise de Sentimentos em tweets sobre a COVID-19 oriundos de cidadãos de 12 países diferentes: EUA, Itália, Espanha, Alemanha, China, França, Reino Unido, Suíça, Bélgica, Holanda, Austrália e Índia. Segundo o estudo, em países como Bélgica, Índia e Austrália houve predominância do sentimento positivo. Já na China, as pessoas majoritariamente apresentaram sentimentos negativos. Entretanto, o trabalho apenas incluiu países da Europa e Ásia, deixando de fora representantes da América do Sul, como o Brasil. Também é importante pontuar que a pesquisa apresentou uma abordagem genérica sobre o tema: no decorrer do trabalho pontos sobre medidas relacionadas a lockdown foram enfatizados, porém os resultados apresentados não especificaram os sentimentos obtidos acerca desse tema.

Convém destacar que não foram encontrados trabalhos de pesquisa que abordassem o tema no contexto brasileiro. O presente trabalho busca, assim, contribuir com o estado da arte ao apresentar uma análise de sentimentos sobre a perspectiva de brasileiros por meio da análise de tweets em Português. Outra contribuição foi a construção de uma base de dados anotados contendo sentimentos relacionados ao lockdown e à COVID-19.

\section{Metodologia}

Este trabalho, em sua perspectiva objetiva, é exploratório na medida em que busca proporcionar maior entendimento sobre um problema e permitir a construção de hipóteses. Quanto a seu paradigma de pesquisa, ele pode ser definido como pragmático, o qual visa, ao invés de encontrar a verdade ou a realidade, facilitar a resolução de problemas humanos, ao buscar a aplicação de "tudo que funciona" para resolver o problema e inclui uma combinação de diferentes estratégias de pesquisa. Consequentemente, o pragmatismo tem uma forte base filosófica nos métodos mistos ou campos de pluralismo metodológico.

Para realização das análises propostas neste trabalho, utilizou-se a técnica de Análise de Sentimento. Foram obtidos dados do Twitter, os quais foram classificados de acordo com seu sentimento utilizando algoritmos de Aprendizado de Máquina. 


\subsection{Coleta e anotação manual dos dados}

Os tweets foram extraídos durante os períodos de 08/05/2020 a 02/06/2020 e 22/06/2020 a 31/07/2020, utilizando a biblioteca Python Tweepy ${ }^{2}$. Ao todo, 38.462 tweets escritos em Português e que continham o termo "lockdown" no seu corpo foram coletados.

Após a extração das mensagens os autores deste trabalho rotularam manualmente 2.100 tweets, de acordo com a polaridade da opinião contida no tweet acerca de lockdown, ou seja, classificando-os em três classes: positiva, negativa e neutra. Optou-se por uma anotação balanceada, garantindo que a base dos dados rotulados contivesse 700 tweets de cada uma das três classes. Como os tweets foram anotados objetivando serem utilizados para treinar os classificadores de Aprendizado de Máquina, a anotação balanceada foi escolhida como forma de não enviesar a classificação. Além disso, cada documento foi classificado por dois anotadores, tendo as divergências sido resolvidas, quando ocorreram, como forma de mitigar possíveis vieses também na anotação.

\subsection{Pré-processamento dos dados}

Para a posterior utilização dos dados coletados, foram necessárias técnicas de préprocessamento. Tendo as bibliotecas NLTK $^{3}$ e scikit-learn ${ }^{4}$ sido utilizadas para esse fim. Primeiramente, todos os tweets foram colocados em minúsculo e realizou-se a tokenização, isto é, a divisão dos textos em tokens, com o auxílio do TweetTokenizer da biblioteca NLTK. Então os tokens passaram por um processo de filtragem, na qual as URLs e os identificadores de usuários foram removidos. Optou-se por manter as hashtags, pois considerou-se que elas poderiam conter informações importantes para a classificação de opinião. Por fim, utilizou-se a técnica TF-IDF para transformar os documentos em vetores de características, etapa necessária para a utilização dos classificadores.

\subsection{Classificação automática dos dados}

Para a classificação automática dos tweets, foi construída uma aplicação de Análise de Sentimento em Python, com o auxílio da biblioteca scikit-learn. Cinco classificadores de Aprendizado de Máquina foram utilizados: Multinomial Nä̈ve Bayes, SVM, Random Forest, uma rede neural MLP e o classificador de Regressão Logística. A escolha dos classificadores se deu pelo fato de os quatro primeiros serem os mais utilizados para mineração de texto com a Língua Portuguesa [Souza et al. 2018], ao passo que o de Regressão Logística também é considerado bastante útil para classificação de texto [Yu et al. 2011].

Os cinco classificadores foram combinados utilizando um Sistema de Múltiplos Classificadores, também conhecido como ensemble, o qual combina classificadores diferentes visando melhorar o desempenho da classificação [Wožniak et al. 2014]. Geralmente, essa combinação alcança resultados melhores que classificadores individuais [Vitório et al. 2019b], então optou-se por utilizá-la como forma de prover uma certeza maior na classificação dos tweets.

Com o mesmo objetivo apontado acima, também foi utilizada a técnica de Active Learning (AL). Esta é uma técnica de Aprendizado de Máquina que consiste na anotação de dados no decorrer da classificação, os quais são utilizados para retreinar o classificador.

\footnotetext{
${ }^{2}$ https://www.tweepy.org

${ }^{3}$ https://www.nltk.org

${ }^{4}$ https://scikit-learn.org/stable/
} 
O método AL usa um conjunto inicial de dados rotulados para o primeiro treinamento do modelo. Então, uma estratégia de seleção é utilizada para solicitar o rótulo de novos dados a fim de atualizar o modelo de aprendizagem. Estes dados são aqueles considerados pela estratégia de seleção mais adequadas para o problema [Zimmermann et al. 2015].

Sendo assim, o ensemble de classificadores, tendo sido treinado com os 2.100 tweets anotados manualmente, foi utilizado para classificar os tweets restantes. O algoritmo, então, solicitava ao usuário o rótulo daqueles tweets para os quais os classificadores tiveram menos certeza da sua classe. Esta estratégia de seleção, que leva em conta a incerteza do classificador, é denominada Uncertainty [Vitório et al. 2019a]. E os tweets cujo rótulo foi fornecido eram adicionados ao modelo, melhorando-o. Neste trabalho, a biblioteca modAL [Danka and Horvath 2018] foi utilizada para implementação da técnica de AL.

Como, nesta pesquisa, lidou-se com um conjunto grande de dados distribuídos temporalmente, os quais são conhecidos como "fluxos contínuos de dados", optou-se pela utilização das duas técnicas supracitadas. Ao se trabalhar com fluxos de dados, é possível se deparar com os chamados opinion drifts: mudanças que podem ocorrer nas opiniões das pessoas acerca de alguma entidade com o passar do tempo [Wang et al. 2013]. E ambas as técnicas utilizadas auxiliam no trato desse problema, melhorando a classificação.

Além disso, como forma de garantir um maior grau de confiança na classificação, foram selecionados apenas os tweets para os quais o ensemble teve uma certeza de pelo menos $60 \%$ para sua classe, além daqueles selecionados pela estratégia de AL. Dessa forma, 13.527 tweets foram considerados nesta pesquisa. Após a anotação automática de todos os tweets, 200 deles foram escolhidos aleatoriamente e anotados manualmente por um dos autores. Com essa anotação, pôde-se estimar a acurácia do classificador: $81 \%$.

\subsection{Identificação das localizações}

Por fim, foi realizada a identificação dos estados brasileiros aos quais pertenciam os autores dos tweets classificados. Sabe-se que o campo localização é de caráter opcional e aberto, logo grande parte dos usuários do Twitter não informa sua localização ou não fornece uma localização válida em seu perfil. Sendo assim, montou-se uma aplicação Python com a ajuda da biblioteca pyUFbr ${ }^{5}$, a qual contém a lista de municípios brasileiros por estado, para identificar automaticamente as localidades.

Após leitura da base de dados, palavras chaves foram identificadas e inseridas manualmente como parâmetros de referência. Por exemplo, as siglas dos aeroportos de algumas capitais brasileiras foram utilizadas, como "SSA" (Salvador, na Bahia), "BSB" (Brasília), "SLZ" (São Luís do Maranhão) e "CWB" (Curitiba-PR). Também fez-se uso de abreviações, como "Jampa", para se referir a João Pessoa, na Paraíba, "Sampa" para São Paulo capital e "BH" para Belo Horizonte, em Minas Gerais. Além de nomes populares de cidades como "Cidade Maravilhosa", para se referir à cidade do Rio de Janeiro, e "Capital do Forró" para Caruaru-PE. Essas nomenclaturas auxiliaram no processo de identificação. Contudo, a dificuldade em obter a geolocalização, bem como a existência de cidades com nomes iguais em estados distintos ocasionaram uma redução do conjunto de dados utilizado para as análises. A Tabela 1 traz exemplos do preenchimento do campo de localização dos usuários do Twitter.

\footnotetext{
${ }^{5}$ https://github.com/Sidon/py-ufbr
} 
Tabela 1. Exemplos do preenchimento do campo de localização e sua localização geográfica correspondente.

\begin{tabular}{l|l|l}
\hline Campo localização & Cidade correspondente & UF \\
\hline "Blumenau, Brasil" & Blumenau & SC \\
\hline "Brasília" & Brasília & DF \\
\hline "ela/dela" & - & - \\
\hline "em casa" & - & - \\
\hline "Fortal city" & Fortaleza & CE \\
\hline "jf" & Juiz de Fora & MG \\
\hline "no meio do mato" & - & - \\
\hline "Nova Iorque, mas a do Maranhão" & Nova Iorque & MA \\
\hline "praia de itapuã, vv" & Vila Velha & ES \\
\hline "recife" & Recife & PE \\
\hline "Rio Grande do Sul, Brasil" & - & RS \\
\hline "rua dos bobos no 0" & - & - \\
\hline "São Bernardo do Campo, Brasil" & São Bernardo do Campo & SP \\
\hline "sp" & - & SP \\
\hline "Ssa-Ba" & Salvador & BA \\
\hline
\end{tabular}

\section{Resultados e Discussão}

Nesta seção, busca-se apresentar resultados que esclareçam os objetivos propostos no inicio deste trabalho. Cabe salientar que os resultados contemplam tweets oriundos das 27 unidades federativas do país. Para realização das análises não foram considerados os tweets com sentimento neutro, os quais representavam $43 \%$ do total coletado. Sendo assim, do montante de 13.527 tweets iniciais, foi utilizada a quantia de 7.757 tweets.

\subsection{Como a população brasileira que emitiu opiniões sobre o lockdown, está reagindo em relação as decisões de implantação da medida?}

De forma geral, no período de estudo, correspondente ao inicio da pandemia, os sentimentos da população brasileira usuária do Twitter e que expressou opiniões sobre o tema em questão foi positivo. Registrou-se um quadro de $58 \%$ de opiniões positivas e $42 \%$ de opiniões negativas. A Figura 1(a), apresenta um gráfico sobre a distribuição dos sentimentos ao longo do tempo, em intervalos de três em três dias, enquanto a Figura 1(b), aborda o crescimento de casos da COVID-19 agrupado neste mesmo intervalo de tempo.

Ao relacionar os gráficos da Figura 1(a) e 1(b), é notório que nos primeiros dias do mês de maio existiu uma oscilação entre sentimentos positivos e negativos. Por esse motivo, não foi possível determinar se o crescimento do número de casos estabeleceu influência neste resultado. Por outro lado, nesse mesmo mês, notou-se que até o dia 23/05, em intervalos que contemplavam pelos menos um dos dias do final de semana (sábado ou domingo), a maioria das opiniões acerca do lockdown eram negativas. Isso pode significar que os usuários ainda estavam preocupados com a impossibilidade de sair de casa em finais de semana durante a vigência do lockdown.

Após esse período, entre os dias 23/05 e 28/06, é possível observar que os sentimentos positivos lideraram de forma constante e com pouca oscilação. Desse modo, ficou claro que o fator "final de semana" já não preocupava tanto os usuários. Nesse período, 
a COVID-19 alcançou picos de 70, 80, e 100 mil casos ${ }^{6}$, o que nos leva a constatar que o crescimento na quantidade de casos passou a ter mais relevância e pode ter causado alguma influência no sentimento dos usuários da rede social. Também, durante o intervalo compreendido entre 16/07 e 27/07, mais uma vez notou-se apenas pequenas alterações no predomínio de sentimentos positivos em relação ao lockdown. E, em meio a esse intervalo, os casos da COVID-19 obtiveram picos de 85 a 100 mil casos influenciando muito provavelmente a opinião dos brasileiros no Twitter.

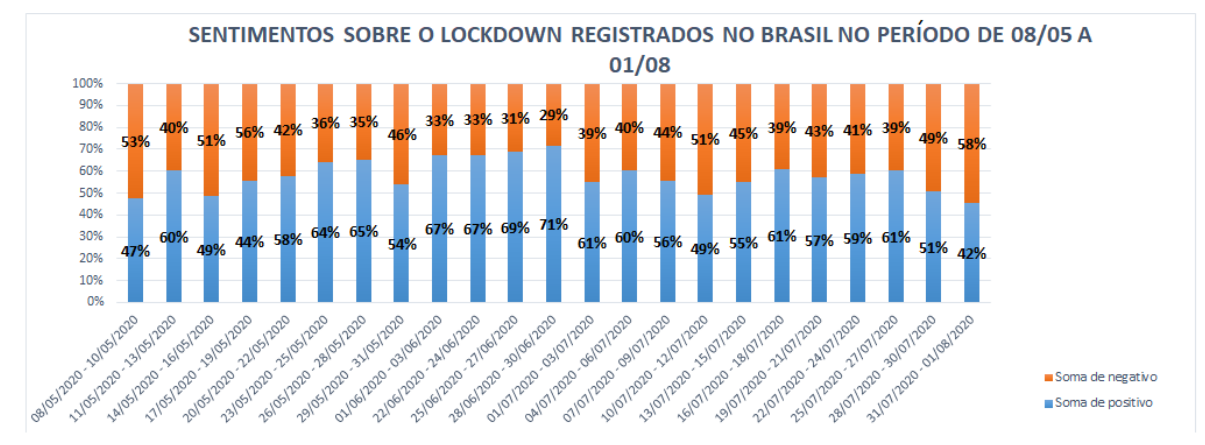

(a)

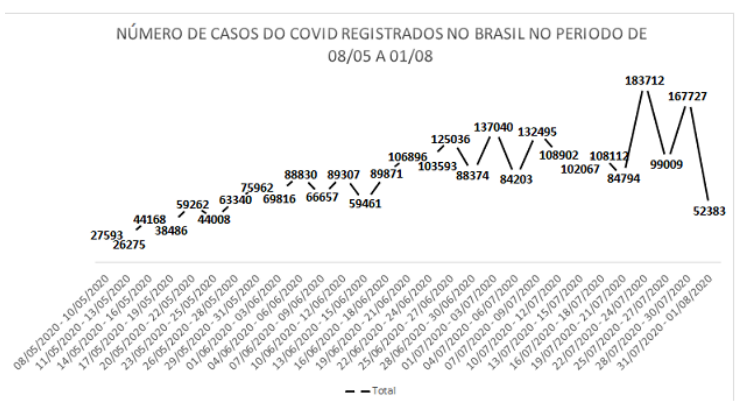

(b)

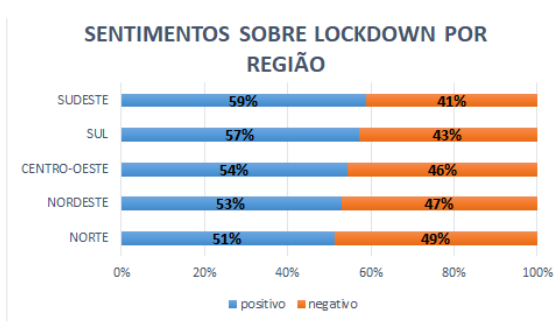

(d)

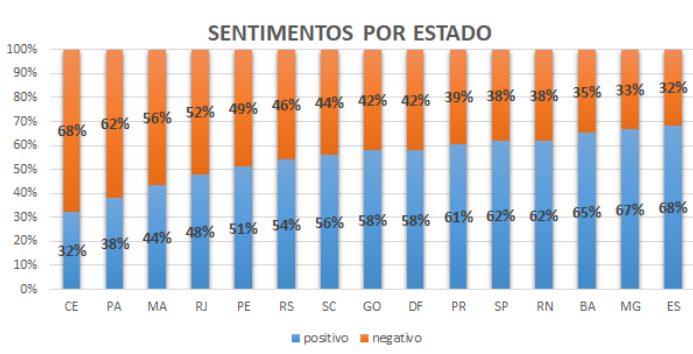

(c)

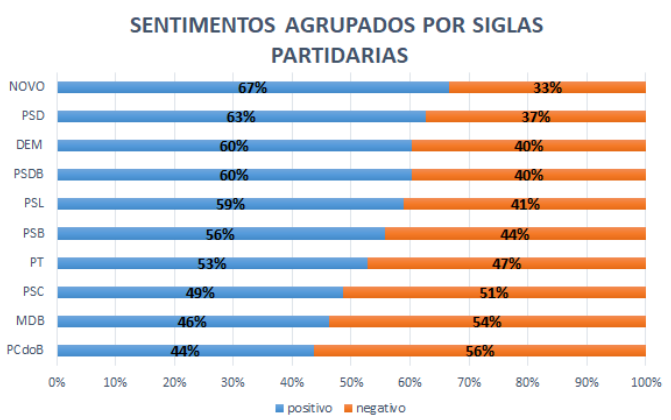

(e)

Figura 1. (a) Distribuição dos sentimentos ao longo do tempo no Brasil; (b) Número de casos da COVID-19 ao longo do tempo no Brasil; (c) Análise de sentimentos por UF; (d) Distribuição dos sentimentos por região do Brasil; (e) Análise de sentimentos por siglas partidária.

\subsection{A localidade, ideologia partidária e riqueza são fatores que influenciaram os sentimentos dos usuários em relação ao lockdown?}

A Figura 1(d) apresenta um gráfico que analisa a distribuição dos sentimentos positivos e negativos por região do Brasil. Vale ressaltar que, devido ao fato de muitos usuários do

\footnotetext{
${ }^{6}$ https://covid.saude.gov.br/
} 
Twitter não informarem localizações válidas em seus perfis, não foi possível identificar a localização de todo o conjunto de dados. Este fator ocasionou a redução no números de tweets analisados. Dos 7.757 tweets validados para as análises, foi possível extrair a localização da origem de 5.874 tweets.

Ao visualizar os resultados da Figura 1(d), torna-se claro que todas as regiões apresentaram superioridade de sentimentos positivos, porém umas em um nível maior que outras. A Região Sul expressou um quadro de sentimentos positivos de 57\%, enquanto a Região Sudeste apresentou o maior percentual de aceitação ao lockdown em relação às demais regiões, com 59\% de sentimentos positivos. Também cabe destacar que esta região contém três dos estados mais populosos do país: São Paulo, Rio de Janeiro e Minas Gerais ${ }^{7}$. A Região Centro-Oeste, por sua vez, obteve $54 \%$ de apoio à medida de lockdown. Já as regiões Nordeste e Norte apresentaram, respectivamente, 53\% e 51\% de sentimentos positivos: os menores valores dentre as cinco regiões. Sendo assim, pode-se considerar que os usuários da região Norte estavam divididos, com um nível de rejeição equiparável ao nível de aceitação.

A Figura 1(c) apresenta um gráfico que explana a distribuição de sentimentos para cada Unidade Federativa (UF). Por sua vez, a Figura 1(e) apresenta os sentimentos agrupados pelas siglas partidárias que governaram as unidades federativas no período de realização do estudo. Ambas as análises foram baseadas no montante nacional de 5.874 tweets, porém, na Figura 1(c) só estão presentes as UFs que possuíam pelo menos 100 tweets classificados como positivo ou negativo. Este recorte se deveu ao fato de que estados com uma quantidade muito baixa de tweets poderiam apresentar uma proporção de sentimentos irreal. Com a análise, notou-se que apenas as UFs do Ceará (CE), Pará (PA), Maranhão (MA) e Rio de Janeiro (RJ) apresentaram superioridade de tweets negativos em relação aos positivos, tendo o $\mathrm{CE}$ apresentado uma rejeição ao lockdown de quase 70\%. Relacionando o gráfico das Figuras 1(c) e 1(e), percebe-se que o sentimento da população em estudo nas unidades federativas supracitadas acompanha o sentimento da sigla partidária que o governa. Ainda vale ressaltar que o cálculo dos sentimentos da sigla partidária foi conduzido com base no agrupamento de todos os estados governados por aquela sigla.

Assim, percebe-se que os usuários habitantes das UFs, em sua grande maioria, sofreram forte influência dos partidos que os governam. Com base no gráfico da Figura 1(e), é visto que partidos como o NOVO, PSD, DEM e PSDB, que apresentam um posicionamento positivo ao lockdown, podem ter induzido a população usuária da plataforma em seus estados a ter um posicionamento semelhante a respeito do assunto. Vale salientar que a predominância de sentimentos negativos no Rio de Janeiro e Pará também pode ter sido decorrente da posição dos partidos PSC e MDB que os governavam, respectivamente. Principalmente no Rio de Janeiro, onde Wilson Witzel, então governador, se mostrou publicamente contra o lockdown ${ }^{8}$. Outro resultado que merece um destaque está relacionado aos estados governados pelo PSL, ex-partido do Presidente da República Jair Bolsonaro, o qual também condenou o lockdown ${ }^{9}$. Tais estados apresentaram mais sentimentos positivos do que as UFs governadas pelo PT, seu principal opositor de esquerda.

\footnotetext{
${ }^{7}$ encurtador.com.br/lvyO5

${ }^{8}$ https://cutt.ly/ckxhsTO

${ }^{9}$ https://cutt.ly/AkxhgVa
} 
Neste contexto, também foi realizada uma observação sobre a análise dos sentimentos agrupando os partidos de acordo com sua ideologia: esquerda ou direita. Para a esquerda foram considerados os partidos PT, PSB e PCdoB, já para direita foram considerados o PSDB, PSC, NOVO, DEM, PSD, PSL e o MDB. É perceptível que no âmbito dos governos das UFs, aquelas governadas por partidos de direita tiveram mais usuários demonstrando na rede social sentimentos positivos em relação às políticas da implantação de lockdown, ao se comparar com aquelas governadas pela esquerda partidária.

Para analisar a influência da riqueza dos estados sobre o sentimento da população, foram tomados como métrica os últimos dados emitidos pelo IBGE sobre o Produto Interno Bruto (PIB) de cada unidade federativa. Mediante essa métrica, foi visto que o ranking de riqueza dos estados não apresentou influência no sentimento dos usuários, uma vez que estados mais pobres ou mais ricos apresentaram porcentagens semelhantes.

Dentro do contexto abordado, ainda é possível associar o número de casos e mortes provocadas pela COVID-19 com a rejeição ou aceitação do lockdown. Esse fator pode ter influenciado no resultado negativo do Mato Grosso do Sul (MS), o qual apresentou uma rejeição ao lockdown de $58 \%$, ao passo que, no período estudado, era uma das UFs com menos casos da doença. Já São Paulo (SP), a UF mais populosa do Brasil, apresentou o maior número de ocorrências e mortes pela doença no país e uma aceitação de $62 \%$ em relação à ideia de lockdown, o que pode levar a crer que o número de casos afeta os sentimentos dos usuários emitentes de opiniões sobre o tema.

\subsection{Análises detalhadas por unidade federativa}

A Figura 2(a) apresenta um gráfico que contém a distribuição dos sentimentos apresentados por usuários do RJ, em um intervalo de três em três dias. Ao relacionar esse gráfico com a taxa de mortes em função da COVID-19, é notório que entre os dias 23/05 e 03/06 existiu uma predominância dos sentimentos positivos, enquanto que, nesse mesmo período, a COVID-19 alcançou picos de 666 a 751 novas mortes. Esse acontecimento nos leva a constatar, mesmo que de forma genérica, que durante esse intervalo de tempo a quantidade de mortes acabou influenciando usuários do Twitter no RJ. Contudo, convém salientar que durante o período de 19/07 a 30/07 os sentimentos negativos em relação ao lockdown ganharam proporção, visto que, mesmo após a implantação do lockdown no estado, o número de novas mortes não havia tido uma redução considerável, levando os usuários a exprimir um sentimento de desaprovação à medida.

O Governo do estado de São Paulo, apesar de não ter adotado a medida de lockdown completamente, empregou diversas outras alternativas semelhantes, como um isolamento social rígido ${ }^{10} \mathrm{e}$ as extensões da quarentena ${ }^{11}$. Dito isto, a análise sobre o lockdown em SP se deu pela expectativa do seu decreto e não pelo seu vivenciamento na prática. Sendo assim, de acordo com a Figura 2(c), é visto que os sentimentos positivos se sobressaíram em relação aos negativos do dia 11/05 até o dia 30/06. Nesse período, também foi observado que houve um grande número de mortes registradas no estado.

A partir do mês de julho é notório que os sentimentos dos usuários em relação ao lockdown foram sofrendo alterações em intervalos de tempo separados. Nos últimos dias os sentimentos dos usuários de São Paulo mostraram uma situação contrária comparada

\footnotetext{
${ }^{10} \mathrm{https}: / /$ cutt.ly/hkxg9QR

${ }^{11} \mathrm{https}: / /$ cutt.ly/ukxg76K
} 


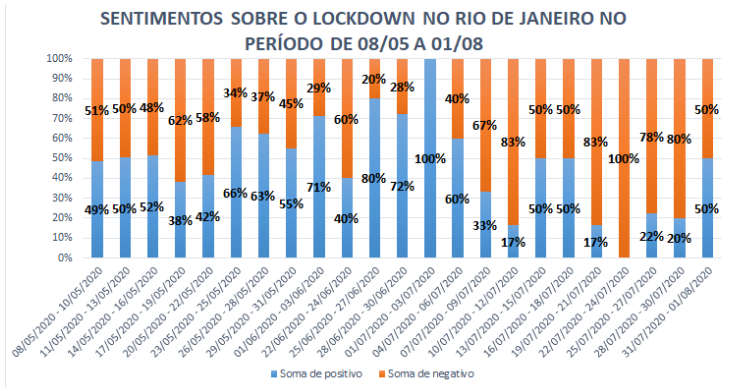

(a)

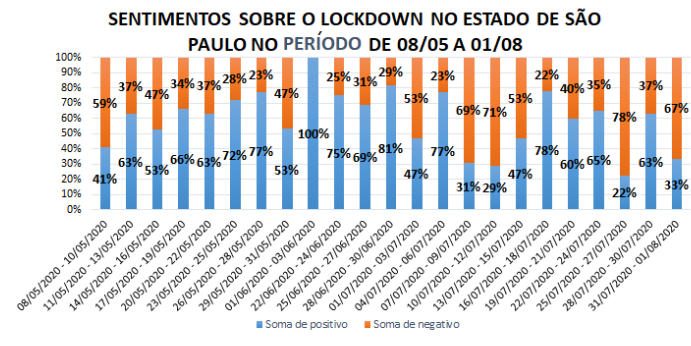

(b)

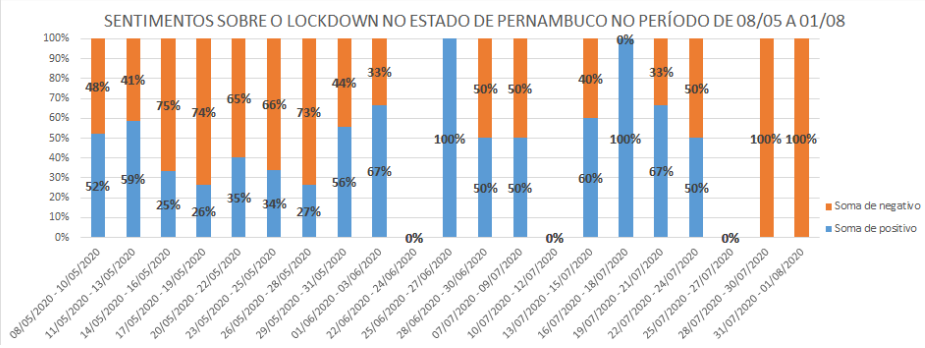

(c)

Figura 2. (a) Distribuição dos sentimentos no estado do Rio de Janeiro; (b) Distribuição dos sentimentos no estado de São Paulo; (c) Distribuição dos sentimentos no estado de Pernambuco.

ao início da pandemia. Os sentimentos negativos atingiram uma porcentagem de $67 \%$ contra o lockdown, e o que antes era tido como um meio para evitar a disseminação do vírus passou a ser visto de outra forma por parte dos usuários da plataforma em São Paulo.

No Maranhão, embora tenha sido o primeiro estado brasileiro a decretar lockdown, foi constatado que não há um padrão relacionando o número de mortes ou o número de casos da COVID-19 com os sentimentos da população usuária da plataforma em relação à medida restritiva. Isto reforça a observação de que o aumento ou a diminuição do número de mortes ou casos pode não ter uma grande interferência nos sentimentos. $O$ Ceará também seguiu a mesma tendência do Maranhão, não apresentando um padrão observável para este estudo.

Pernambuco, por sua vez, em virtude do aumento no número de mortes que ultrapassava a marca de 1 mil, teve seus primeiros indícios de lockdown por volta do dia $11 / 05^{12}$, por meio de uma iniciativa do governo do estado que reforçou as medidas de isolamento social para evitar a disseminação acelerada da doença. A princípio, a iniciativa por parte do governo em tornar a quarentena naquele momento mais rígida para o bem da população gerou sentimentos positivos. O decreto impedia as pessoas de saírem de casa e andar pelas ruas, exceto para trabalhar em serviços essenciais ou realizar atividades indispensáveis, como comprar alimentos ou retirar benefícios sociais em lotéricas e bancos. O decreto ainda contemplava o rodízio de veículos em cinco municípios do estado. Entretanto, a predominância entre sentimentos positivos negativos variou ao longo do tempo. Entre os dias 14/05 e 28/05, houve uma maior quantidade de mensagens com sentimentos negativos. Entre os dias 29/05 e 27/06, por sua vez, o sentimento positivo tornou a ser aquele mais identificado entre as mensagens. Já no final do período analisado,

\footnotetext{
${ }^{12} \mathrm{https} / / / \mathrm{cutt} . l y / 2 \mathrm{kxhwlI}$
} 
as mensagens criticando e rejeitando o lockdown se sobrepuseram mais uma vez.

\subsection{Nuvens de palavras}

Com auxilio das bibliotecas WordCloud ${ }^{13}$, pandas $^{14}$ e matplotlib ${ }^{15}$, ambas do Python, um script foi elaborado para gerar a imagem da nuvem de palavras. Para isso, o conjunto de dados referente aos tweets positivos e negativos foram processados individualmente.

A Figura 3 apresenta as nuvens de palavras obtidas quando os usuários se referiam de forma positiva e negativa ao lockdown. Ao observar a nuvem associada aos sentimentos positivos, nota-se que a mesma comporta palavras de apoio, como "favor", quando os usuários relatavam que eram a favor do lockdown, como por exemplo na frase: "eu sou super a favor do lockdown por aqui com direito a multa". O uso das palavras "necessário", "precisa" e "deveria" quando visualizavam a necessidade da medida, como expresso na frase: "Eu não acho ruim um lockdown na cidade, apesar de afetar meu empreendimento. Eu acho necessário e apoio". E, palavras como "agora" , "decreta logo" e "finalmente" quando desejavam que o lockdown fosse adotado naquele momento, tal como na frase: "Finalmente lockdown aqui na cidade amém".

Palavras como "amor" também foram encontradas de forma isolada, porém expressando uma emoção de euforia e revolta. Pode-se entender o seu uso como parte da expressão "pelo amor de Deus", como na frase:"impõe lockdown nessa cidade logo pelo amor de Deus", provavelmente pelo fato de o lockdown ainda não ter sido implementado no estado/município. O nome do governador de São Paulo, João Dória, representado pelos termos "Doria" e "jdoriajr", também obteve destaque na nuvem. Essa presença pode ter sido ocasionada pela hesitação do governador em decretar a medida ${ }^{16}$, a qual era desejada pela maioria do público.

Já analisando os sentimentos negativos, a junção das palavras "Especialistas alemães" se destacou na nuvem, bem como o termo "alemães dizem". Muitos usuários utilizaram uma matéria de um jornal alemão criticando o lockdown que foi implantado naquele país para se posicionar contra a medida no Brasil. As palavras "desemprego" e "fome" também estão presentes na nuvem negativa. É possível que os usuários acreditassem que, com a implantação do lockdown, a economia, e principalmente o setor de comércio, seriam severamente afetados [Fernandes 2020]. Com o seu fechamento, o índice de desemprego aumentaria e por consequência muitos usuários se queixaram da possibilidade de fome ou da escassez de alimentos para a suas famílias. A palavra " $d i$ tadura" também apareceu, cuja ocorrência pode ser explicada pelo fato de os usuários compararem o lockdown a um regime totalitário. Por fim, a aparição do nome do presidente Jair Bolsonaro (pelo termo "jairbolsonaro") na nuvem se dá por ele ter se posicionado contrário à medida, conforme citado anteriormente, e desta forma acabar exercendo influência sobre a população e usuários que o apoiam.

\section{Considerações Finais}

Neste trabalho, foi realizada uma Análise de Sentimentos acerca da implantação da medida restritiva denominada lockdown no Brasil. Mediante os resultados, pôde-se perceber

\footnotetext{
${ }^{13}$ encurtador.com.br/aFZ14

${ }^{14} \mathrm{https}: / /$ pandas.pydata.org

${ }^{15} \mathrm{https} / / /$ matplotlib.org

${ }^{16}$ https://cutt.ly/Fkxhy25
} 


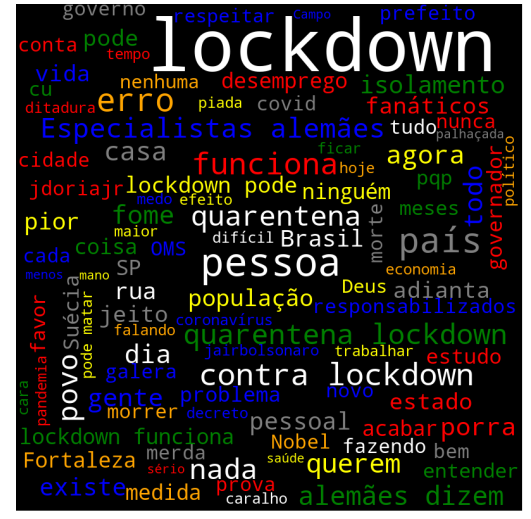

Nuvem de palavras obtida através dos tweets com sentimentos negativos

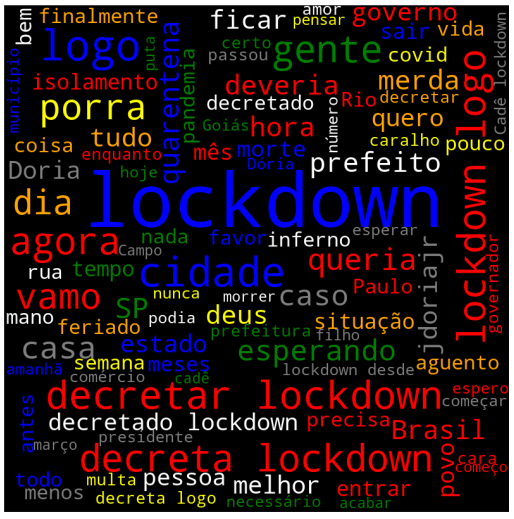

Nuvem de palavras obtida através dos tweets com sentimentos positivos.

Figura 3. Nuvens de palavras obtidas através dos tweets.

que a maioria dos usuários brasileiros da rede social Twitter que emitiram mensagens sobre o tema (58\%) reagiram positivamente a sua adoção, tendo em vista as classificações obtidas no período de tempo do estudo.

Durante a pesquisa, não foi possível determinar a predominância de apenas um fator sobre as opiniões em questão, ficando claro que elas variavam com base em diversos aspectos. Já, no que diz respeito a questões de escopo, a pesquisa buscou demonstrar avaliações partindo do ponto de vista mais genérico, como visto nas demonstrações por regiões e país como um todo, até chegar em uma avaliação mais individual por estado ou federação. Neste sentido, é notável que a maioria das federações ou estados abordados, possuem seus próprios fatores de influência, sejam eles partidários, geográficos, econômicos ou ideológicos.

Ainda cabe pontuar que o presente trabalho provê uma base de dados de sentimentos do Twitter, anotados de acordo com sua polaridade, e por conseguinte contribui como referência para pesquisas futuras que objetivem comparar resultados acerca do tema. Também é possível que gestores se alimentem dessa pesquisa para avaliar a tomada de novas decisões em relação à pandemia de COVID-19. Para trabalhos futuros, é de fundamental importância evoluir a pesquisa de modo a deixa-la mais sólida no que se refere a realização de inferências estatísticas. Além disso, é pautável explorar através das técnicas de análise de sentimentos, esse ou outros temas relacionados ou não à pandemia, que causam impacto na opinião pública.

\section{Referências}

Alswaidan, N. and Menai, M. E. B. (2020). A survey of state-of-the-art approaches for emotion recognition in text. Knowledge and Information Systems, 62(8):2937-2987.

Bai, H. M., Zaid, A., Catrin, S., Ahmed, K., and Ahmed, A. (2020). The socio-economic implications of the coronavirus pandemic (covid-19): A review. Int. J. Surg., 8(4).

Barkur, G., Vibha, and Kamath, G. (2020). Sentiment analysis of nationwide lockdown due to covid 19 outbreak: Evidence from india. Asian Journal of Psychiatry, 51.

Carvalho, M. S., Lima, L. D. d., and Coeli, C. M. (2020). Ciência em tempos de pandemia. Cadernos de São Paulo, 36. 
Chehal, D., Gupta, P., and Gulati, P. (2020). Covid-19 pandemic lockdown: An emotional health perspective of indians on twitter. International Journal of Social Psychiatry, 0(0):0020764020940741.

Danka, T. and Horvath, P. (2018). modAL: A modular active learning framework for Python. available on arXiv at https://arxiv.org/abs/1805.00979.

Dubey, A. D. (2020). Twitter sentiment analysis during covid19 outbreak. Available at SSRN 3572023.

Fernandes, N. (2020). Economic effects of coronavirus outbreak (covid-19) on the world economy. Available at SSRN 3557504.

Kaur, R. and Ranjan, S. (2020). Sentiment analysis of 21 dayscovid-19 indian lockdown tweets.

Liu, B. and Zhang, L. (2012). A Survey of Opinion Mining and Sentiment Analysis, pages 415-463. Springer US, Boston, MA, EUA.

Moreira, R. d. S. (2020). Covid-19: unidades de terapia intensiva, ventiladores mecânicos e perfis latentes de mortalidade associados à letalidade no brasil. Cadernos de Saúde Pública, 36:e00080020.

Pastor, C. K. (2020). Sentiment analysis of filipinos and effects of extreme community quarantine due to coronavirus (covid-19) pandemic. Available at SSRN 3574385.

Souza, E., Costa, D., Castro, D. W., Vitório, D., Teles, I., Almeida, R., Alves, T., Oliveira, A. L. I., and Gusmão, C. (2018). Characterising text mining: a systematic mapping review of the portuguese language. IET Software, 12(2):49-75.

Vitório, D., Souza, E., and Oliveira, A. L. I. (2019a). Evaluating active learning sampling strategies for opinion mining in brazilian politics corpora. In 19th EPIA Conference on Artificial Intelligence, pages 695-707, Cham. Springer.

Vitório, D., Souza, E., and Oliveira, A. L. I. (2019b). Using active learning sampling strategies for ensemble generation on opinion mining. In 8th Brazilian Conference on Intelligent Systems (BRACIS), pages 114-119. IEEE.

Wang, D., Feng, S., Wang, D., and Yu, G. (2013). Detecting opinion drift from chinese web comments based on sentiment distribution computing. In Web Information Systems Engineering - WISE 2013, pages 72-81.

Watson, R., Corbett, J., Galletta, D. F., Ives, B., Mandviwalla, M., and Tremblay, M. (2020). Covid-19 and is: Challenges and opportunities for people, careers, and institutions. In Proceedings of AMCIS 2020.

Wožniak, M., Graña, M., and Corchado, E. (2014). A survey of multiple classifier systems as hybrid systems. Information Fusion, 16:3 - 17.

Xavier, A. R., Silva, J. S., Almeida, J., Conceição, J. F. F., Lacerda, G. S., and Kanaan, S. (2020). Covid-19: manifestações clínicas e laboratoriais na infecção pelo novo coronavírus. J Bras Patol Med Lab, 56:1-9.

Yu, H.-F., Huang, F.-L., and Lin, C.-J. (2011). Dual coordinate descent methods for logistic regression and maximum entropy models. Machine Learning, 85(1-2):41-75. 
Yue, L., Chen, W., Li, X., Zuo, W., and Yin, M. (2018). A survey of sentiment analysis in social media. Knowledge and Information Systems, 60(2):617-663.

Zimmermann, M., Ntoutsi, E., and Spiliopoulou, M. (2015). Incremental active opinion learning over a stream of opinionated documents. arXiv preprint arXiv:1509.01288. 